\title{
Original
}

\section{Comparison of Angiogenesis in Bone Defect Healing Process due to the Difference in the Frequency of Low-Intensity Pulsed Ultrasound (LIPUS)}

\author{
Hodaka Sasaki ${ }^{1,2)}$, Kazuya Monden ${ }^{1)}$, Masao Yoshinari' ${ }^{2)}$ and Yasutomo Yajima ${ }^{1,2)}$ \\ 1) Department of Oral and Maxillofacial Implantology, Tokyo Dental College, Tokyo, Japan \\ 2) Oral Health Science Center, Tokyo Dental College, Tokyo, Japan \\ (Accepted for publication, February 4, 2016)
}

\begin{abstract}
Low-intensity pulsed ultrasound (LIPUS) is known to promote bone defect healing and also angiogenesis. It was reported the frequency of the LIPUS was related to directivity and the depth of penetration, but the differences in angiogenesis during bone defected healing remains unknown. The aim of this study is to investigate the effect of low and high frequency LIPUS exposure for the angiogenesis during rat femur bone defect healing process by molecular biological and histomorphological evaluations. Bone defects of $1.6 \mathrm{~mm}$ in diameter were created in both femurs of ten-week-old male Long-Evans rats $(n=30)$. Right femur as LIPUS exposure groups were exposed LIPUS (intensity: $30 \mathrm{~mW} / \mathrm{cm} 2$, burst width: $200 \mu \mathrm{s}$, time: $15 \mathrm{~min} / \mathrm{day}$ ) and divided into a low frequency (1.5 MHz, L15) group and a high frequency (3.0 MHz, L30) group. Left femur as non-exposed LIPUS group were used as control. After 3, 5, and 7 days, femurs were removed and quantitative RT-PCR (qRT-PCR) for vascular endothelial growth factor (VEGF), histomorphological and immunohistochemical evaluations and measurement of new formed capillary vessel ratio in bone defected are were conducted. The results of qRT-PCR were indicated that $V E G F$ expression of L15 at 5 days was significantly higher than that of L30 and control group $(p<0.05)$. Immune-positive reaction of VEGF was recognized in fibroblasts, endothelial cells, periosteal cells and osteoblast and these expression in LIPUS exposure groups were stronger than control groups. The capillary vessel formation ratio in upper layer of bone defected area in L15 group was significantly increased compared to L30 and control group at 7 days $(p<0.05)$. In conclusion, 1.5 MHz frequency of LIPUS exposure was more effective to promote VEGF expression and angiogenesis than 3.0 $\mathrm{MHz}$ in rat femur bone defected healing.
\end{abstract}

Key words: Low-intensity pulsed ultrasound, Frequency, Bone healing, Angiogenesis, VEGF

\section{Introduction}

Recently, dental implants are now being widely used for prosthodontic treatment for missing teeth ${ }^{1)}$, and these also become to treat for patients with implant risk factors such as osteoporosis and diabetes. Therefore, a number of studies has been reported on improving of implant body surface topography and chemistry for shortening the period of establish osseointegration, and increase the success rate of implant in these patients ${ }^{2-4)}$. On the other hands, some studies were reported about methods of improving the jaw bone as host side. One of them were known to inject drugs such as bone morphogenetic protein-2 $2^{5)}$, fibroblast growth factor $2^{6)}$ and simvastatin $^{7)}$ into the extraction socket, and they were shown to promote bone healing. However, these methods were containing the risk of toxicity by overdose and the limitation of area for keeping the drug. Therefore, that low-intensity pulsed ultrasound (LIPUS), a non-invasive technique that causes no drug-related

Correspondence to: Dr. Hodaka Sasaki, Department of Oral and Maxillofacial Implantology, Tokyo Dental College, 2-9-18 Misakicho, Chiyoda-ku, Tokyo, 101-0061 Japan; Phone: +81-3-63809257; E-mail: hosasaki@tdc.ac.jp side effects and utilizes physical stimulation seems very useful method of improving the jaw bone.

LIPUS was still used in clinical settings to promote healing of normal ${ }^{8)}$ and intractable ${ }^{9,10)}$ bone fractures in the field of orthopedic surgery. In basic research, it was reported osteoblast cell line were maturated and accelerated calcification by LIPUS stimulation $^{11-13)}$. Furthermore, it was clarified LIPUS exposure promotes healing process and increases bone mineral density by rat femurs fracture model ${ }^{14,15}$. It was also reported about the effect of LIPUS exposure for bone with titanium implant. LIPUS improved the contact rate of newly formed bone in implants placed in rabbit femurs ${ }^{16)}$ and promotes the formation of new bone tissue in areas of bone augmentation in the rabbit maxillary sinus ${ }^{17}$. These results indicate that LIPUS is useful method for implant therapy, because it seems to promote achieving osseointegration and extraction socket healing process.

Three major parameters of LIPUS exposure were known as intensity, exposure time and frequency. The frequency of LIPUS was especially important for using dental implant treatment, because it was known to contribute to directivity and the depth 
of penetration. Higher frequencies of LIPUS improve the directivity of ultrasonic energy and decrease the depth of penetration ${ }^{18)}$. In the bone healing process, the difference of LIPUS intensity was known to contribute to osteocyte and osteoblast differentiation, and optimal parameter is defined ${ }^{11,19}$. On the other hand, the effect of differences in the frequency of LIPUS during bone healing was still not clarified. In the previous study ${ }^{20)}$, high $(3.0 \mathrm{MHz})$ and low $(1.5 \mathrm{MHz})$ frequency of LIPUS exposure groups were promoted rat femur bone defect healing compared to the nonLIPUS exposure group. But there was no significant differences between both high and low frequency of LIPUS exposure group in the evaluation for the bone maturation. However, it was recognized the regression area of blood clots in the low frequency group was deeper than high frequency group at the early stage of bone defect healing process. We hypothesized, this regression of blood clots by LIPUS exposure was occurred by increasing capillary vessel, and the difference of LIPUS frequency influenced the angiogenesis during the bone healing process.

The aim of this study is to investigate the effect of low and high frequency LIPUS exposure for the angiogenesis during rat femur bone defect healing process by molecular biological and histomorphological evaluations.

\section{Materials and Methods Surgical procedure and LIPUS irradiation}

Ten-week-old male Long-Evans rats (Sankyo Labo Service Corporation, INC, Tokyo, Japan; $\mathrm{n}=30$ ) were used in this study. After peritoneal injections of pentobarbital sodium (Somnopentyl ${ }^{\circledR}$ $0.9 \mu \mathrm{l} / \mathrm{g}$, Kyoritsu Seiyaku Corporation, Tokyo, Japan) were administered as general anesthesia. For surgery, the hind legs of the rats were shaved considerably and the outside skin of the distal femur was incised longitudinally, and the femur was exposed by stripping the periosteum. The bone defects were created in both femurs at $3 \mathrm{~mm}$ from the articular surface of the knee joint using a round bur (1.6 mm diameter). The depth of the bone defect was created to reach the opposite side of the cortical bone. After the bone defect was created, the periosteum was replaced and the surgical wound sutured. Starting from one day after bone defect creation, the bone defect area of the right femur was transcutaneously exposed to LIPUS (intensity: $30 \mathrm{~mW} / \mathrm{cm}^{2}$, burst width: $200 \mu \mathrm{s}$, time: $15 \mathrm{~min} /$ day, transducer size: $\mathrm{M}[3.2 \mathrm{~cm}$ diameter], frequency: $1.5 \mathrm{MHz}$ or $3.0 \mathrm{MHz}$ ) with gel as a conductive medium using ST-SONIC (Ito Co, Ltd, Tokyo, Japan). The frequency parameters for LIPUS were low frequency $(1.5$ $\mathrm{MHz}$; L15) and high frequency (3.0 MHz; L30) and LISUS exposure period was set to $3,5,7$ days ( $n=10$ for each days). The left femurs that composed the non-LIPUS group were used as the control (Cont) group (Fig. 1). After 3, 5 and 7 days LIPUS exposure, rats were sacrificed and both sides of femur were harvested. Two samples for each day of L15, L30 and control groups were used for quantitative RT-PCR and remaining three samples were used for immunohistochemical staining. All experiments were performed according to the Guidelines for the Treatment of Animals at Tokyo Dental College (approval ID: 253002).

\section{RNA extraction and quantitative RT-PCR (qRT-PCR)}

The rats were sacrificed with pentobarbital sodium after 3, 5 and 7 days, and bone tissue samples for RNA extraction was collected using trephine bar $(2.8 \mathrm{~mm}$ internal diameter, Micro Tech Corp, Tokyo, Japan) from the center of the bone defect area $(n=2$ for each days). Collected bone tissue was kept in RNAlater RNA stabilization reagent (Applied Biosystems) and then homogenized (tungsten carbide beads; $5 \mathrm{~mm}$ diameter, $28 \mathrm{~Hz}, 2 \mathrm{~min}$ ) using a Tissue Lyser (QIAGEN). Total RNA was extracted from the lysate using an RNeasy ${ }^{\circledR}$ Mini kit (QIAGEN) according to the manufacturer's protocol and quantified with a NanoDrop ${ }^{\circledR}$ Spectrophotometer ND-1000 (NanoDrop Technologies, Wilmington, DE, USA). The mRNA expression levels of VEGF (AssayID Rn01511601_m1) were confirmed by qRT-PCR using a TaqMan ${ }^{\circledR}$ MGB probe (Applied Biosystems) and normalized against $\beta$-actin (Applied Biosystems). Total RNA was reversetranscribed using QuantiTect ${ }^{\circledR}$ Reverse Transcription (QIAGEN), and qRT-PCR was performed with TaqMan ${ }^{\circledR}$ Fast Universal PCR Master Mix (Applied Biosystems) and an ABI 7500 Fast Prism Sequence Detection System (Applied Biosystems) according to the manufacturer's instructions. This quantification for each sample was duplicated, and results were analyzed using the $\Delta \Delta \mathrm{Ct}$ method. Values are expressed as the mean and standard error and were analyzed with the Tukey's test $(\mathrm{p}<0.05)$.

\section{Sectioning and staining for histomorophological and immunohistochemical evaluation}

Samples were fixed in $10 \%$ neutral buffered formalin for 7 days and decalcified with EDTA (pH 7.0-7.5; Wako Pure Chemical Industries, Osaka, Japan) at room temperature over 6 days. Paraffin sections of $3 \mu \mathrm{m}$ in thickness were prepared and deparaffinized with xylene and rehydrated in ethanol. Hematoxylin-eosin (HE) stains for histomorphological evaluation was performed by standard protocol. Sections for immunohistochemical (IHC) stains were then washed in $10 \mathrm{nmol} / 1$ phosphate-buffered saline (PBS, $\mathrm{pH}$ : 7.4) and immersed in $0.3 \%$ hydrogen peroxide in ethanol for 30 min to block endogenous peroxidase activity. After the sections were washed in PBS, they were blocked with $3 \%$ normal bovine serum (BSA; Roche Applied Science, Indianapolis, USA). After reacting the sections with the primary antibodies, rabbit antiVEGF (Abcam, Cambridge, UK; diluted 1:200), for 1 hour at room temperature, they were reacted with the secondary antibody, biotinylated anti-rabbit IgG antibody (Histofine MAX-PO [MULTI]; Nichirei, Toyo, Japan), for 30 minutes at room temperature. After washing in PBS, the sections were stained 
Hodaka Sasaki et al.: LIPUS induces angiogenesis on bone healing

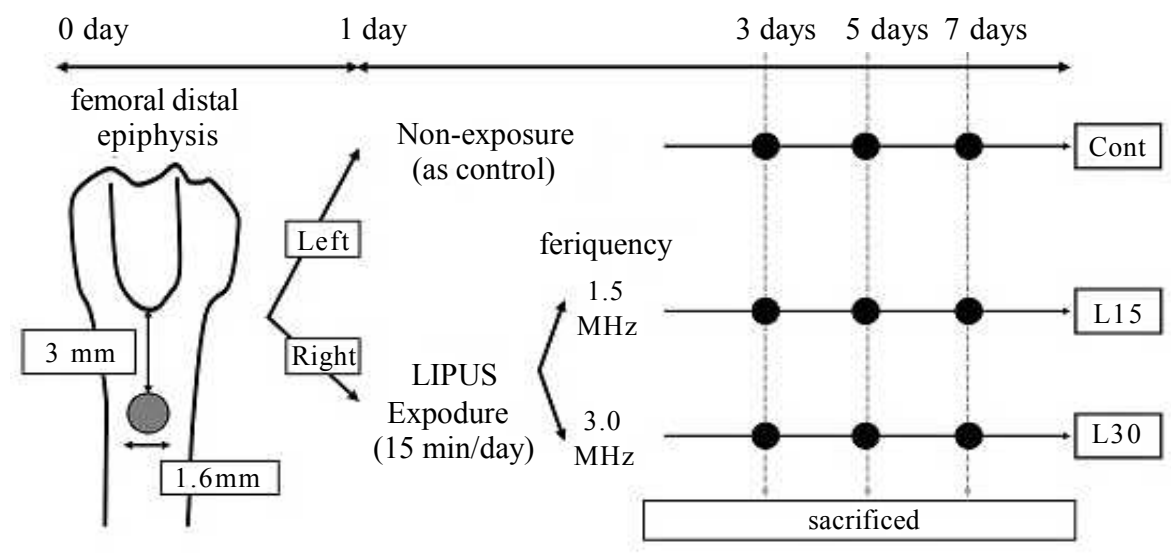

Figure 1. Bone defect model in rat femur and experimental protocol

(a)

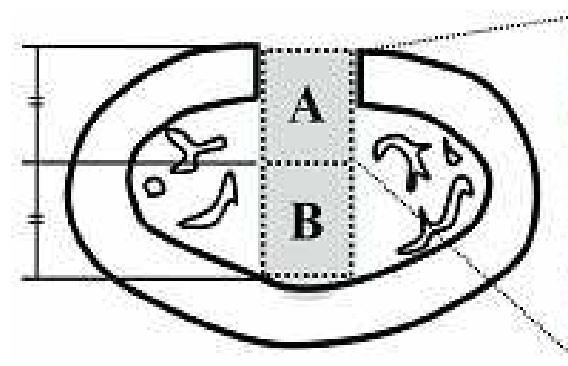

(b)

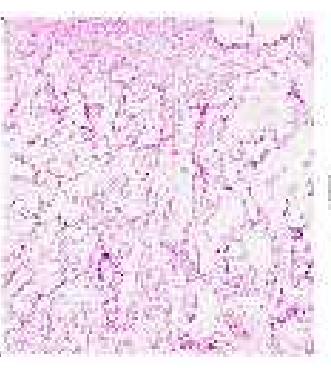

(c)

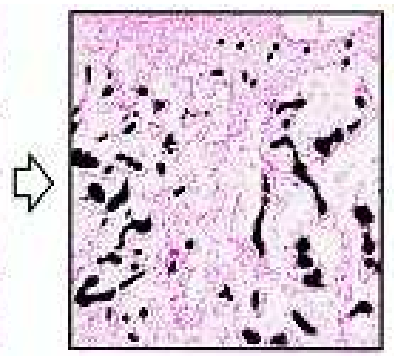

(d)

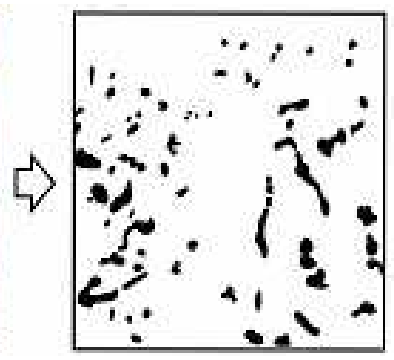

Figure 2. Scheme of regions of interest (ROI) for measuring capillary vessel formation area.

(a) ROI: Upper layer area (A) and lower layer area (B) in bone defected area; (b) Extracted ROI image from HE section; (c) Visualization of capillary vessel formation area; (d) Extracted visualization of capillary vessel formation area

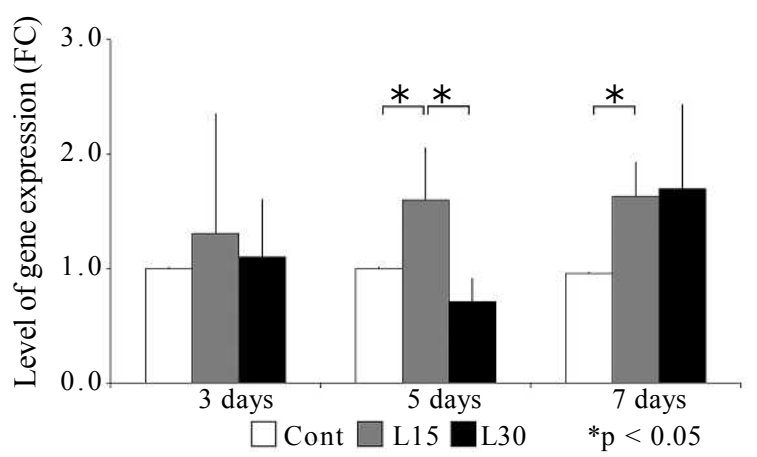

Figure 3. Gene expression quantification for VEGF by quantitative RT-PCR at 3, 7 and 10 days. FC: Fold Change

with 3,3'-diaminobenzidine (DAB)(DAB substrate kit Nichirei, Tokyo, Japan) at room temperature. After counterstaining with a hematoxylin solution, they were dehydrated and enclosed according to the established protocol, and then were observed with a universal photo microscope (Axiophot 2, Carl Zeiss, Oberkochen, Germany).

Measurement of new formed capillary vessel in bone defect area

The region of interest (ROI) for measuring capillary vessel formation was defined in the bone defected area and it was divided into upper and lower layer area (Fig. 2). The image of immunohistochemical staining sections were captured using a universal photo microscope (Axiophot 2). Visualization of capillary vessel formation area was performed by Adobe Photoshop (Adobe Systems, San Jose, USA). The number of pixels of capillary vessel formation area and bone defected area was counted using ImageJ software (National Institutes of Health, Bethesda, Maryland, USA). The ratio of new formed capillary area was calculated by pixels of new formed capillary area divided by pixels of bone defected area. The Mann-Whitney Utest was used for the statistical analysis $(p<0.05)$.

\section{Results}

\section{Gene expression pattern of VEGF in bone defected area}

The gene expression of vascular endothelial growth factor $(V E G F)$ was quantified using samples harvested from bone defected area after 3, 5 and 7 days (Fig.3). No significant difference in gene expression of $V E G F$ was observed in samples taken from 3 days after bone defect creation. The level of VEGF expression in L15 group was significantly higher than control and L30 group at 5 days ( $\mathrm{p}<0.05$ ). Moreover, $V E G F$ expression in L15 group control 

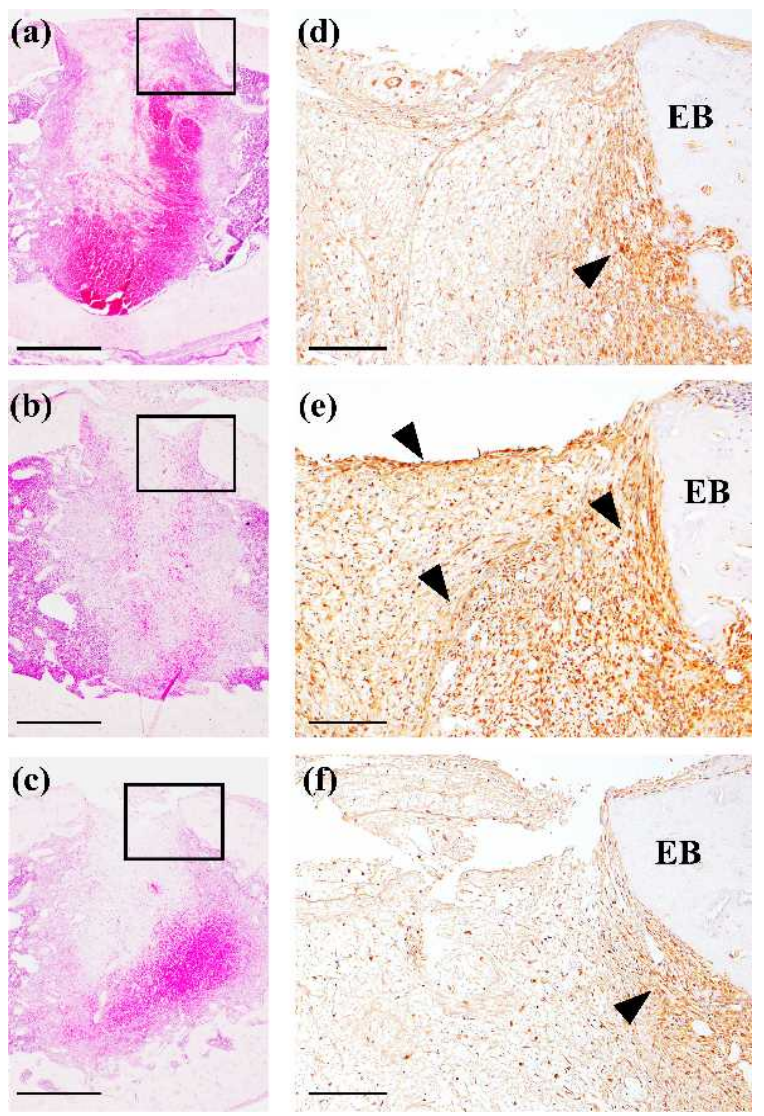

Figure 4. HE and IHC for VEGF at 3 days.

upper column: Cont, middle column: L15, lower column: L30 (a),(b),(c): low magnification of bone defected area (HE), Bar: $0.5 \mathrm{~mm}$; (d),(e),(f): high magnification of upper layer of bone defected area (IHC), Bar: $0.2 \mathrm{~mm}$; EB: existing bone, Arrow: positive reaction

group was significantly increased compared to at 7 days after surgery $(\mathrm{p}<0.05)$.

\section{Histomorphological evaluation and immunohistochemical localization of VEGF in bone defect area}

Low magnification images of hematoxylin and eosin staining for histomorphological evaluation and high magnification images of immunohistochemical staining of VEGF in bone defected area were shown in Figs. 4, 5 and 6.

At 3 days after surgery, blood clots were observed in all layers of the bone defect area in the control group (Fig. 4a). Whereas, blood clot retraction was observed in both LIPUS exposure groups and that of area in L15 group (Fig. 4b) was larger and deeper than the L30 group (Fig. 4c). The strong immune-positive reaction of VEGF in L15 group was recognized in fibroblasts, endothelial cells and periosteal cells adjacent to stump of existed bone in bone defected area (Fig. 4e). On the other hands, immunoreaction of VEGF in control and L30 group were observed at inner periosteum cells but there was no and/or weak reaction in bone defected area (Fig. 4d, f).
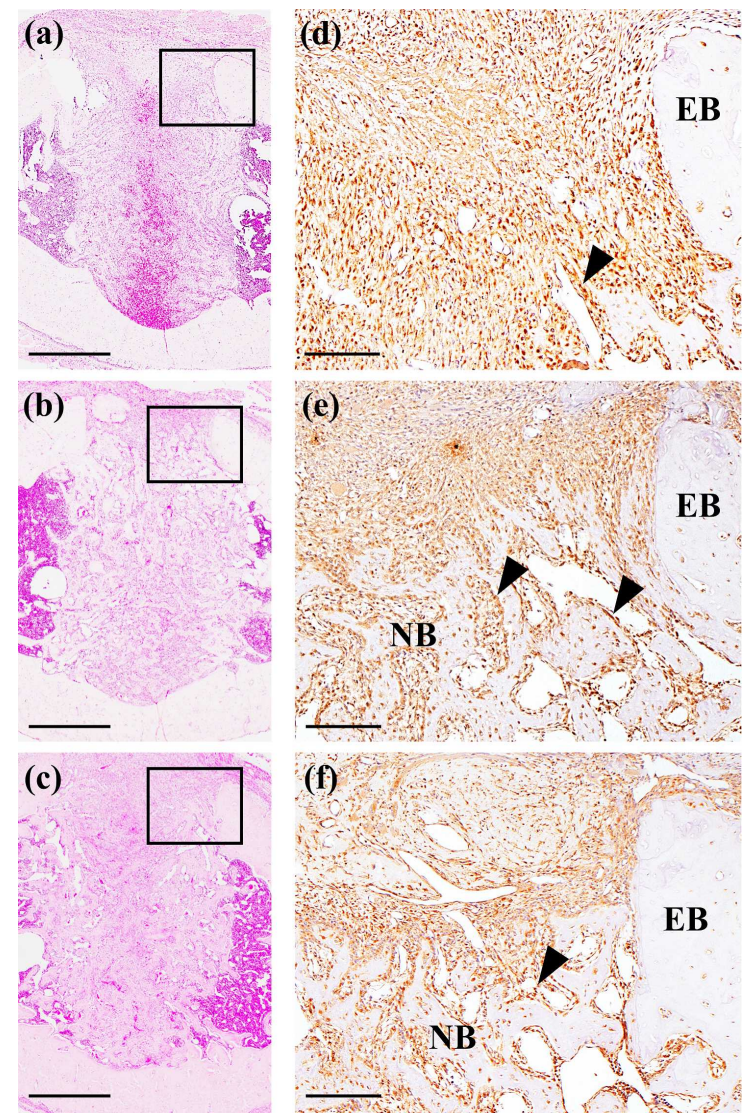

Figure 5. HE and IHC for VEGF at 5 days.

upper column: Cont, middle column: L15, lower column: L30 (a),(b),(c): low magnification of bone defected area (HE), Bar: $0.5 \mathrm{~mm}$; (d), (e),(f): high magnification of upper layer of bone defected area (IHC), Bar: $0.2 \mathrm{~mm}$; EB: existing bone, NB: newly formed bone, Arrow: positive reaction

At 5 days after surgery, blood clots was still remained at center of bone defected area in control groups (Fig. 5a), whereas bone decocted area of LIPUS exposure groups were filled with newly formed bone (Fig. 5b, c). The positive immunoreaction of VEGF was recognized in osteoblast adjacent to newly formed bone and vascular endothelial cells of all 3 groups. The upper layer of bone defected area capillary in LIPUS exposure groups (Fig. 5e, f) were more dilated than that of control group (Fig. 5d), and more strong immunoreaction in endothelial cell was recognized in L15 group than L30 group.

At 7 days after surgery, cancellous bone maturation was observed in all layers of the bone defect area in all 3 groups (Fig. 6a-c). In the upper layer of bone defected area, newly bone and capillary formation in cortical bone defected area was recognized in both LIPUS exposure groups and immune-reaction of VEGF was observed in endothelial cells and outer periosteum cells (Fig. $6 \mathrm{e}, \mathrm{f})$, and newly formed cortical bone in L15 groups was more dense than L30 group. Furthermore, few newly formed bone and capillary was seen in cortical bone area in control group and positive reaction pattern of VEGF was similar to that of 5 days. In the 
Hodaka Sasaki et al.: LIPUS induces angiogenesis on bone healing
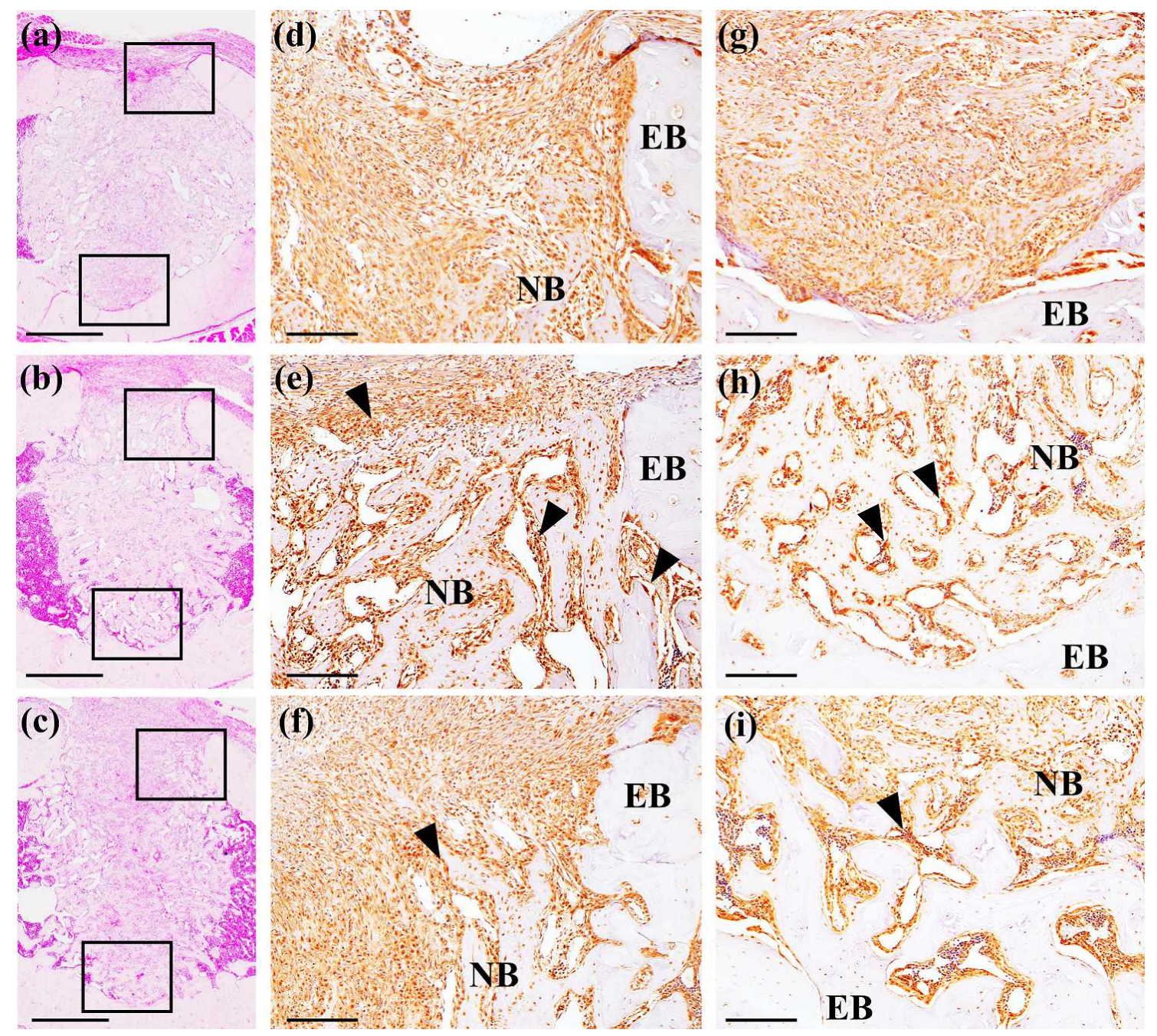

Figure 6. HE and IHC for VEGF at 7 days.

upper column: Cont, middle column: L15, lower column: L30

(a), (b), (c): low magnification of bone defected area (H-E), Bar: $0.5 \mathrm{~mm}$; (d), (e), (f): high magnification of upper layer of bone defected area (IHC), Bar: $0.2 \mathrm{~mm}$; (g), (h), (i): high magnification of lower layer of boned defected area (IHC), Bar: 0.2 $\mathrm{mm}$; EB: existing bone, NB: newly formed bone, Arrow: positive reaction

lower layer of cancellous bone defected area was also observed newly formed bone and capillary in both LIPUS exposure groups (Fig. 6h, i). On the hands, weak positive reaction was recognized in osteoblast adjacent to few newly bone.

\section{Measurement of capillary vessel formation ratio in upper and lower layer of bone defected area}

The ratio of capillary vessel formation in upper and lower bone defected area was shown in Fig. 7. There was no significant difference among all 3 groups in the both upper and lower layer of capillary formation ratio at 3 and 5 days after surgery. On the other hands, significant increasing ratio of capillary formation area was recognized to compare with control group in both layers, and L30 group in Upper layer at 7day after surgery $(\mathrm{p}<0.05)$.

\section{Discussion}

The effects of different frequencies of LIPUS exposure for angiogenesis during bone healing process in rat femur bone defect models were investigated by molecular biological and histomorphological evaluations.

LIPUS is a type of ultrasound energy that passes through living tissues ${ }^{18)}$ and is known to promote healing of fractures and bone defects ${ }^{8,14}$. The parameters for LIPUS include intensity, exposure time and frequency. LIPUS intensity was known to relate the osteoblast proliferation and we used intensity of $30 \mathrm{~mW} / \mathrm{cm}^{2}$ LIPUS in this study. Because, previous studies reported an intensity of $30 \mathrm{~mW} / \mathrm{cm}^{2}$ LIPUS promotes osteoblast differentiation in vitro ${ }^{13)}$ and promotes fracture healing in a rat femur model in vivo ${ }^{14)}$. Furthermore, the United States Food and Drug Administration currently recommends that an intensity of $30 \mathrm{~mW} /$ $\mathrm{cm}^{2}$ be used when using LIPUS for human bone fracture.

LIPUS exposure is known not only to accelerate osteoblastic 


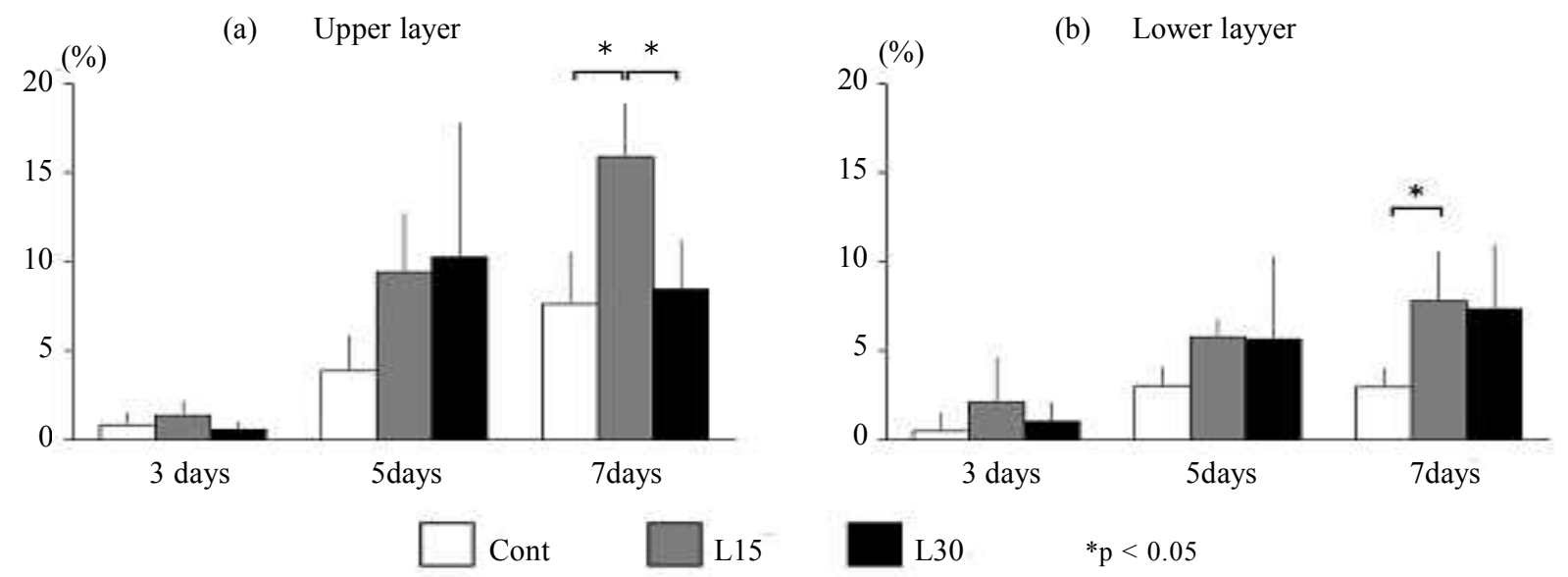

Figure 7. Capillary vessel formation ratio in upper and lower layer of bone defected area at 3,5 and 7 days.

cell maturation and bone fracture healing but also angiogenesis ${ }^{21)}$. Angiogenesis is key component for wound healing and tissue regeneration. The process of bone healing divided into 4 stages; hematoma formation stage, fibrocartilaginous callus formation stage, bony callus formation stage, and bone remodeling stage. Angiogenesis is seems to promote the transition into the bone remodeling stage from hematoma formation stage by creating new blood vessels which bring oxygen, serve inflammatory cells and bone precursor cells to the injury site. In this experiment, the regression of blood clot was observed in both LIPUS exposure groups (L15 and L30) at 3 days. Furthermore, LIPUS exposure group had higher new capirally vessel formation ratio in all layer of bone defected area at 7 days than control group ( $p<0.05$, data not shown).

VEGF known as a key growth factor directly associated with angiogenesis and neovascularization is produced by endothelial cells, macrophages, fibroblast, smooth muscle cells and osteoblasts $^{22}$. Some studies reported $V E G F$ expression was upregulated by LIUPS exposure. The $1 \mathrm{MHz}$ frequency of ultrasound stimulated expression of VEGF in human osteoblast, gingival fibroblast and monocyte in vitro ${ }^{23}$. William et al. ${ }^{24)}$ described immunohisitochemical positive reaction of VEGF was increased with $1.5 \mathrm{MHz}$ frequency of LIPUS exposure at 4 weeks compared to controls in unilateral rabbit ulna defect filled with bone graft substitute model. In this experiment, immunepositive reaction of VEGF was recognized in fibroblasts, endothelial cells, periosteal cells and osteoblast and these expression in LIPUS exposure groups were stronger than control groups. From these results, it was considered that LIPUS exposure promoted gene expression of $V E G F$ during bone defect healing by activating fibroblasts, endothelial cells and pre-osteoblast in periosteal.

LIPUS frequency is known to contribute to directivity and the depth of penetration. The frequency of LIPUS for healing has a range of $0.75-3 \mathrm{MHz}$, with most machines set at a frequency of
1 or $3 \mathrm{MHz}^{18)}$ Lower frequency of LIUPS have greater depth of penetration, but less direction. From this characters, a lower frequency $(1 \mathrm{MHz})$ of LIPUS was recommended to use for deeper injuries and/or patient with more subcutaneous fat ${ }^{18)}$. There was few study to investigate the effect of LIPUS with difference frequency on bone healing. Takebe et al. ${ }^{17)}$ reported low frequency $(1 \mathrm{MHz})$ of LIPUS group was recognized more new bone growth than control group and high frequency $(3 \mathrm{MHz})$ of LIPUS group in rabbit sinus augmentation model. On the other hand, our previous study concluded gene expression of bone differentiation markers (osteopontin and osteocalcin) and volume of newly formed bone are in rat femur bone defected area had no significant difference between L15 and L30 groups ${ }^{20}$. On the other hands, there was significant difference was recognized in gene expression of $V E G F$ at 5 days and capillary vessel formation ratio in upper layer of bone defected area at 7 days between L15 and L30 group. Reher et al. ${ }^{23)}$ were investigated to compare the protein expression of VEGF in osteoblast and monocyte between $0.45 \mathrm{MHz}$ an $1 \mathrm{MHz}$ frequency of LIPUS exposure, and they concluded the optimum intensities were 15 to $30 \mathrm{~mW} / \mathrm{cm} 2$ (SATA) with $0.45 \mathrm{MHz}$ of ultrasound and 0.1 to $0.4 \mathrm{~W} / \mathrm{cm}^{2}$ (SATA) with $1 \mathrm{MHz}$ of ultrasound. Moreover, half-value depth the distance at which $50 \%$ of the ultrasound energy has been absorbed by the tissue is known to relate with frequency of ultrasound. It was reported half- value depth of $1 \mathrm{MHz}$ frequency was approximately 2.3 to $5 \mathrm{~cm}$, and that of $3 \mathrm{MHz}$ was 0.8 to $1.6 \mathrm{~cm}^{25}$. From these results, we suggested intensity of $30 \mathrm{~mW} / \mathrm{cm}^{2}$ used in this experiment is too low to up-regulate VEGF expression in this experiment model. But, it seems to be difficult to use a higher intensity with $3 \mathrm{Mhz}$ frequency of LIUPS, because intensity of LIPUS is defined as the range of intensity 0.5 to $50 \mathrm{~mW} / \mathrm{cm}^{2}$. In addition, it was reported $100 \mathrm{~mW} / \mathrm{cm}^{2}$ statement value was the upper limits in the safety statement made by the American Institute of Ultrasound in Medicine (AIUM ${ }^{26)}$. In the case of LIPUS exposure for human extracted socket, these effects need to reach deeper area than this 


\section{Hodaka Sasaki et al.: LIPUS induces angiogenesis on bone healing}

animal model. Therefore, $1 \mathrm{MHz}$ is the recommended frequency to promote angiogenesis by LIPUS exposure during bone defected healing.

In conclusion, 1.5 MHz frequency of LIPUS exposure was more effective to promote VEGF expression and angiogenesis than $3.0 \mathrm{MHz}$ in rat femur bone defected healing.

\section{Conflict of Interest}

The authors have declared that no COI exists.

\section{References}

1. Dierens M, Vandeweghe S, Kisch J, Nilner K and De Bruyn H. Long-term follow-up of turned single implants placed in periodontally healthy patients after 16-22 years radiographic and peri-implant outcome. Clin Oral Implants Res 23: 197-204, 2012

2. Fischer K and Stenberg T. Prospective 10-year cohort study based on a randomized controlled trial (RCT) on implant-supported full-arch maxillary prostheses. Part 1: sandblasted and acid-etched implants and mucosal tissue. Clin Implant Dent Relat Res 14: 808815,2012

3. Buser DA, Schroeder A, Sutter F and Lang NP. The new concept of ITI hollow- cylinder and hollow-screw implants: Part 2. Clinical aspects, indications, and early clinical results. Int J Oral Maxillofac Implants 3: 173-181, 1988

4. Omar OM, Lennerås ME, Suska F, Emanuelsson L, Hall JM, Palmquist A and Thomsen P. The correlation between gene expression of proinflammatory markers and bone formation during osseointegration with titanium implants. Biomaterials 32: 374-386, 2011

5. Fiorellini JP, Howell TH, Cochran D, Malmquist J, Lilly LC, Spagnoli D, Toljanic J, Jones A and Nevins M. Randomized study evaluating recombinant human bone morphogenetic protein-2 for extraction socket augmentation. J Periodontol 76: 605-613, 2005

6. Lalani Z, Wong M, Brey EM, Mikos AG, Duke PJ, Miller MJ, Johnston C and Montufar-Solis D. Spatial and temporal localization of FGF-2 and VEGF in healing tooth extraction sockets in a rabbit model. J Oral Maxillofac Surg 63: 1500-1508, 2005

7. Wu Z, Liu C, Zang G and Sun H. The effect of simvastatin on remodeling of the alveolar bone following tooth extraction. Int J Oral Maxillofac Surg 37: 170-176, 2008

8. Heckman JD, Ryaby JP, McCabe J, Frey JJ and Kilcoyne RF. Acceleration of tibial fracture-healing by non-invasive, low-intensity pulsed ultrasound. J Bone Joint Surg Am 76: 26-34, 1994

9. Lerner A, Stein H and Soudry M. Compound highenergy limb fractures with delayed union: our experience with adjuvant ultrasound stimulation (exogen). Ultrasonics 42: 915-917, 2004

10. Rutten S, Nolte PA, Korstjens CM, van Duin MA and Klein-Nulend J. Low-intensity pulsed ultrasound increases bone volume, osteoid thickness and mineral apposition rate in the area of fracture healing in patients with a delayed union of the osteotomized fibula. Bone 43: 348-354, 2008

11. Gleizal A, Li S, Pialat JB and Beziat JL. Transcriptional expression of calvarial bone after treatment with low-intensity ultrasound: an in vitro study. Ultrasound Med Biol 32: 1569-1574, 2006

12. Sena K, Leven RM, Mazhar K, Sumner DR and Virdi AS. Early gene response to low-intensity pulsed ultrasound in rat osteoblastic cells. Ultrasound Med Biol 31: 703-708, 2005

13. Suzuki A, Takayama T, Suzuki N, Sato M, Fukuda T and Ito K. Daily low-intensity pulsed ultrasoundmediated osteogenic differentiation in rat osteoblasts. Acta Biochim Biophys Sin (Shanghai) 41: 108-115, 2009

14. Wang SJ, Lewallen DG, Bolander ME, Chao EY, Ilstrup DM and Greenleaf JF. Low intensity ultrasound treatment increases strength in a rat femoral fracture model. J Orthop Res 12: 40-47, 1994

15. Warden SJ, Fuchs RK, Kessler CK, Avin KG, Cardinal RE and Stewart RL. Ultrasound produced by a conventional therapeutic ultrasound unit accelerates fracture repair. Phys Ther 86: 1118-1127, 2006

16. Liu Q, Liu X, Liu B, Hu K, Zhou X and Ding Y. The effect of low-intensity pulsed ultrasound on the osseointegration of titanium dental implants. $\mathrm{Br} \mathrm{J}$ Oral Maxillofac Surg 50: 244-250, 2012

17. Takebe H, Nakanishi Y, Hirose Y and Ochi M. Effect of low intensity pulsed ultrasound stimulation on sinus augmentation in rabbits. Clin Oral Implants Res 25: 735-741, 2014

18. Speed CA. Therapeutic ultrasound in soft tissue lesions. Rheumatology (Oxford) 40: 1331-1336, 2001

19. Angle SR, Sena K, Sumner DR and Virdi AS. Osteogenic differentiation of rat bone marrow stromal cells by various intensities of low-intensity pulsed ultrasound. Ultrasonics 51: 281-288, 2011

20. Monden K, Sasaki H, Yoshinari M and Yajima Y. Effect of low-intensity pulsed ultrasound (LIPUS) 
J.Hard Tissue Biology Vol. 25(2):157 -164, 2016

with different frequency on bone defect healing. $J$ Hard Tissue Biol 24: 189-198, 2015

21. Padilla F, Puts R, Vico L and Raum K. Stimulation of bone repair with ultrasound: a review of the possible mechanic effects. Ultrasonics 54: 1125-1145, 2014

22. Beamer B, Hettrich C and Lane J. Vascular endothelial growth factor: an essential component of angiogenesis and fracture healing. HSS J 6: 8594, 2010

23. Reher P, Doan N, Bradnock B, Meghji S and Harris M. Effect of ultrasound on the production of IL-8, basic FGF and VEGF. Cytokine 11: 416-423, 1999

24. Walsh WR, Langdown AJ, Auld JW, Stephens P, Yu Y, Vizesi F, Bruce WJ and Pounder N. Effect of low intensity pulsed ultrasound on healing of an ulna defect filled with a bone graft substitute. J Biomed Mater Res B Appl Biomater 86: 74-81, 2008

25. Draper DO, Castel JC, and Castel D. Rate of temperature increase in human muscle during 1 $\mathrm{MHz}$ and $3 \mathrm{MHz}$ continuous ultrasound. J Orthop Sports Phys Ther 22: 142-150, 1995

26. Miller DL. Safety assurance in obstetrical ultrasound. Semin Ultrasound CT MR 29: 156-164, 2008 